ADLFI. Archéologie de la France -

\title{
Vallée de la Dronne
}

\section{Christian Chevillot}

\section{OpenEdition}

Journals

Édition électronique

URL : http://journals.openedition.org/adlfi/2787

ISSN : 2114-0502

Éditeur

Ministère de la culture

Référence électronique

Christian Chevillot, "Vallée de la Dronne », ADLFI. Archéologie de la France - Informations [En ligne], Aquitaine, mis en ligne le 01 mars 2008, consulté le 01 mai 2019. URL : http://

journals.openedition.org/adlfi/2787

Ce document a été généré automatiquement le 1 mai 2019.

(c) Ministère de la Culture et de la Communication, CNRS 


\title{
Vallée de la Dronne
}

\author{
Christian Chevillot
}

Identifiant de l'opération archéologique : 025311

Date de l'opération : 2008 (PI)

\section{VALLÉE DE L'ISLE - LA DOUBLE - BERGERACOIS}

1 Nous avons poursuivi au cours de l'année 2008 notre prospection-inventaire dans la haute vallée de la Dronne et plus particulièrement dans le triangle Lisle - Saint-Pardoux-laRivière - Thiviers.

2 Comme d'habitude, nous avons étendu cette recherche en vallée de l'Isle, dans la Double et nous avons eu connaissance de découvertes en vallée de la Vézère et de la Dordogne. Le travail de terrain de l'année 2008 a été marqué par des découvertes significatives :

- une prospection au sol pour identifier la sépulture para-mégalithique de Tabaterie - Sendougne à La Gonterie-Boulouneix. Cette sépulture néolithique avait été repérée en 1984 par Emile Neau, qui nous a légué le mobilier recueilli alors, par la découverte de plusieurs sites gallo-romains inédits, par la découverte de plusieurs militaria d'époque romaine qui ont donné lieu à publication ;

- par la connaissance de la découverte en 2006 d'un superbe manche de patère en bronze, en cours d'étude ;

6 - enfin, l'identification de nouveaux cluzeaux dans la région de Mussidan.

\section{VALLÉE DE LA DRONNE ET SES AFFLUENTS}

Paléolithique 
La densité d'occupation au cours du Paléolithique moyen est toujours aussi nette, et bien moindre au Paléolithique supérieur :

- sites de Bourliou (Bussac), du Baconnet (Valeuil), du Châtenet (Lisle), de La Chauprade (Tocane), des Roches (Brantôme), du Suquet et de l'Etang (Cercles);

- petits sites du Paléolithique supérieur des Grèzes (Saint-Just), ou des Brandissoux (Goût-Rossignol).

\section{Néolithique}

Cette période est toujours aussi bien documentée car les sites sont nombreux. Nouveaux fragments d'anneaux-disques en roches vertes, haches polies en roches dures ou en silex avec industrie abondante (grattoirs, armatures de flèches, etc.) aux Fargeix (L'Isle), La Monerie-Les Champs et Aux Maynes (Montagrier), Beauséjour-Bressols (Tocane), les Roches (Brantôme), Saint-Laurent-de-Gogabaud (Condat-sur-Trincou), la Terre-Rouge et Prés Cheysas (Champagnac-de-Bélair), etc.

Mais la plus intéressante redécouverte reste celle de la sépulture dolménique de Tabaterie-Sendougne, repérée en 1984 par Émile Neau. Grâce à René Ventenat, nous avons pu en retrouver l'emplacement précis. C'est une sépulture collective du Néolithique récent ou final, aménagée dans un chaos de pierres tombées de la falaise. Plusieurs aménagements internes sont observables, notamment de larges dalles soigneusement juxtaposées verticalement pour en colmater un des côtés. Émile Neau avait alors recueilli dans des terres remuées par les blaireaux les restes de quatre à cinq individus (adultes et enfants) et un ciseau aménagé dans un canon de cervidé.

\section{Âge du Bronze}

Le site de Prés Cheysas, à Champagnac-de-Bélair, a encore livré sur deux petites zones très charbonneuses (fosses probables) de nombreux tessons de céramiques du Bronze Final IIIb, auxquels étaient associés quelques silex et un petit peson encoché pour lester un filet de pêche.

\section{Âge du Fer}

Le site de "Aux Maines » à Montagrier a livré de l'amphore Dr. Ia et Ib. Le site de La Rigale à Villetoureix a encore donné une abondante série d'amphores vinaires italiques Dr. Ia et Ib et de la céramique commune datable entre 120 et $50 \mathrm{av}$. J.-C. (La Tène D). Le site de Saint-Martial-de-Ribérac a aussi livré des tessons de céramiques communes de la Tène D2 et des fragments d'amphores italiques vinaires (Dr. Ia et Ib).

\section{Période gallo-romaine}

Les prospections menées en 2008 ont permis de découvrir et recenser de nouveaux sites et les récoltes y ont été abondantes.

Le site de "Aux Maines" (Montagrier) a livré à divers prospecteurs de la céramique commune. À La Veyssière (Mensignac), petit site en fond de vallée, sur la commune de Tocane-Saint-Âpre, près de Beauséjour, un important site gallo-romain a livré du mobilier allant du I ${ }^{\mathrm{er}}$ s. au IV $\mathrm{e}$ s. : amphores (Dr. 20, Pascual I, Dr. 2-4), du verre, une serpe en fer et des tessons de sigillée et de céramiques communes ; sur le site de La Rigale (Villetoureix), nous avons récolté un as de Claude, une fibule de type pseudo La Tène II en bronze, de l'os, de la sigillée des $\mathrm{I}^{\mathrm{er}} \mathrm{s}$. et $\mathrm{II}^{\mathrm{e}} \mathrm{s}$., du verre, des tessons d'amphores et de la céramique commune en abondance; à Saint-Martial-de-Ribérac, près du cimetière, une vaste villaa livré un arc de fibule en bronze, de l'amphore, du verre, de la sigillée et de la céramique 
commune ; au lieu-dit «Les Chenevières », sur la commune de Petit-Bersac, l'installation du lagunage de la station d'épuration, en août 2006, a permis d'observer un fossé parallèle à la Dronne, d'environ $1,5 \mathrm{~m}$ de large, en forme de $\mathrm{V}$, dans lequel se trouvaient de nombreux ossements de chevaux (sanctuaire ?). Non loin de là il a été recueilli un superbe manche de patère en bronze à tête de bélier ; sur le site de Prés Cheysas (Champagnac-deBélair), la villagallo-romaine a livré deux militaria; des travaux d'aménagement d'un lagunage à Quinsac sur le site du stade, ont détruits une partie du site gallo-romain: arases de murs, restes de sols en place, etc. ; commune de Cercles, derrière l'église, il a été repéré un site gallo-romain du Haut-Empire ; aux Mouyauds (La Tour-Blanche), vestiges d'un petit site gallo-romain; à Villeneuve (Cherval), nous avons repris la prospection de cette importante villaqui s'étend sur plusieurs hectares $\left(\mathrm{e}^{\mathrm{er}} \mathrm{s}\right.$. au V $\mathrm{e}$ s.). Les marbres de diverses origines y sont particulièrement abondants. Il a été récolté : un as d'Agrippa, un billon de Tétricus $\mathrm{I}^{\text {er }}$, un petit militaria, des clous, des huîtres, de la faune, de petits tessons de sigillée, 1 tesson en céramique vernissée de St-Rémy-en-Rollat, de la paroi fine, de la commune, de la DS.P., du verre, des fragments de meules.

\section{VALLÉE DE L'ISLE ET SES AFFLUENTS}

\section{Paléolithique}

Sur la commune de Chancelade, au lieu-dit « Lavaure », non loin de l'abri de Reymonden, des travaux de nettoyage de la falaise, ont fait apparaitre une grotte qui était cachée par la végétation, et des carrières, pour la pierre et pour des meules. La grotte a hélas été vidée anciennement, mais nous y avons récolté quelques silex taillés, témoins de son occupation au Paléolithique. Plusieurs sites du Paléolithique moyen ont été repérés : les Barres (Château-l'Évêque), à Agonac au lieu-dit Les Gannes-La Porcherie, à BrochardGrassaval à St-Front-d'Alemps, à Las Groulièras (Coursac), à Bourbou (Agonac), à Perbouyer et au Civadaud (St-Médard-de-Mussidan), etc.

\section{Néolithique}

À Lespinasse (Chancelade), les prospections de la villagallo-romaine ont permis de récolter une petite industrie néolithique; à La Sauvagie (Château-l'Évêque), une hache polie a été découverte dans le mur d'une ancienne maison; à Bourbou (Agonac), petit site néolithique ; à Las Groulièras (Coursac), présence de silex taillés néolithiques, ainsi qu'à Grassaval-Brochard (St-Front-d'Alemps) ; au lieu-dit le Sorbier (St-Laurent-sur-Manoire), haches polies; à Peyrignolle et au Pont-Rouge (Grignols), petits sites néolithiques; à Perbouyer et au Civadaud (St-Médard-de-Mussidan), deux sites néolithiques dont le dernier étendu et riche en mobilier: haches polies, haches taillées, flèches, grattoirs, outils divers et meules ; enfin, de l'autre côté de l'Isle à La Roche (St-Martin-l'Astier), petit site néolithique.

\section{Âge du Bronze}

Sur la pente est d'Ecornebœuf (Coulounieix-Chamiers), face à la ville de Périgueux, S. Mongibeaux a noté la présence d'un vaste épandage de tessons de céramiques du Bronze Final IIIa et IIIb surtout, avec des formes et des décors typiques ainsi qu'un fragment de chenet en terre cuite et d'une fusaïole.

\section{La Tène}


Toujours à Ecornebœuf (Coulounieix-Chamiers), ce même épandage a aussi livré de la céramique de La Tène D2 et des fragments d'amphores Dr. I. À Chancelade, sur la villade Lespinasse, présence de tessons de La Tène D et d'amphores vinaires italiques Dr. I. À Las Groulièra», près du Cerf-du-Meymie à Coursac : amphores Dressel Ia et Ib et céramique commune de La Tène D1 et D2. gallo-romain coupé par la route et en partie détruit par un lotissement récent. Nombreux moellons, mortier, tegulae, imbrices et un mobilier peu abondant: amphores PascualI, céramique commune, morceaux de verres, rares et minuscules tessons de sigillée, le tout datable du Haut-Empire.

\section{tegulaeet pieds d'amphores Pascual I.}

À Périgueux, dans le jardin des Arènes, T. Laval a recueilli dans les terres des massifs de fleurs, un fragment de tuile canal avec un graffitod'époque médiévale. Rue du Puits (Mussidan), sur la petite ligne de falaise en rive gauche de la Crempse, J.-C. Héseault et D. Massoulier, ont exploré cette falaise, qui présente de nombreuses galeries. Dans un remblai ancien, ils ont recueilli de nombreux tessons d'un grand bugeadier en pâte rouge. Sur la même commune, un peu plus en amont, à « La Frénie Haut », ils ont topographié un souterrain aménagé inédit chez $\mathrm{M}$. Muller; le souterrain présente deux salles dont une avec de curieuses arcades taillées dans la paroi en molasse tendre et un puits de $14 \mathrm{~m}$ de profondeur. 


\section{MEULIÈRES}

\section{INDEX}

Index géographique : Aquitaine, Dordogne (24), Lisle, Saint-Pardoux-la-Rivière, Thiviers Thèmes : amphore italique, céramique du bronze, céramique du fer, cervidé, dolmen, fibule, grotte, industrie lithique, inventaire, mobilier, monnaie, ossement animal, patère, pêche, sépulture, sépulture collective, verre, villa Index chronologique : âge du Bronze, âge du Fer, Empire romain, Moyen Âge, Néolithique, Paléolithique, Temps Modernes

operation Prospection inventaire (PI)

\section{AUTEURS}

CHRISTIAN CHEVILLOT

BEN 